\title{
Improved Low Power Multichannel Pulse Amplitude Analyzer
}

\author{
Jin Yang*, Maolin Xiong, Xu Zhao \\ Chengdu University of Technology, Chengdu, China \\ Email: *605514589@qq.com
}

How to cite this paper: Yang, J., Xiong, M.L. and Zhao, X. (2020) Improved Low Power Multichannel Pulse Amplitude Analyzer. Open Access Library Journal, 7: e6629.

https://doi.org/10.4236/oalib.1106629

Received: July 18, 2020

Accepted: August 10, 2020

Published: August 13, 2020

Copyright $\odot 2020$ by author(s) and Open Access Library Inc.

This work is licensed under the Creative Commons Attribution International License (CC BY 4.0).

http://creativecommons.org/licenses/by/4.0/

\begin{abstract}
Multichannel pulse amplitude analyzer is the core part of ray energy spectrum analysis system. In order to meet the requirements of ultra-low power consumption and portable high accuracy of the instrument, this design is characterized by the application of differential circuit in the screening circuit, which eliminates the analog switch, capacitance and diode, and greatly reduces the power consumption of the instrument. Without the use of diodes, circuits can discriminate pulses of any magnitude, especially in gamma-ray spectrometer systems. The overall structure is $89 \mathrm{C} 52 \mathrm{MCU}$ as the control core, simple design, external RAM storage measurement data, through RS-232 serial port and computer communication. According to the requirements of the measured frequency, switching values of $R$ and $C$ can be applied to a variety of energy spectrum analyzer systems.
\end{abstract}

\section{Subject Areas}

Nuclear Physics

\section{Keywords}

Multi-Channel Pulse Amplitude Analyzer, Energy Spectrum, Portable, Ultra-Low Power Consumption

\section{Introduction}

Multichannel pulse amplitude analyzer (MCA), as an important part of radioactivity measurement instrument, is the basis of subsequent ray energy spectrum data processing. With the advent of the era of intelligence and people's concern for their own natural environment, it is an inevitable demand for the development of nuclear technology industry to carry out research on intelligent, small, low-power and highly applicable multichannel pulse amplitude analyzer. 
At present, the development of portable MCA is relatively backward. High power consumption, difficult development and poor expansion performance are the main factors limiting its application scope. In energy spectrum measurement, the amplitude of the detector's output pulse signal is proportional to the energy of the incident particle, and the number of radiation times of the particle of a certain energy level in unit time reflects the radiation probability of the particle of that energy level. By measuring the amplitude and frequency of pulse signal, the relationship between the occurrence probability and energy of incident particles can be obtained. Therefore, the nuclear spectrum data acquisition system with multi-channel pulse amplitude analyzer as the core is the key of nuclear radiation detection technology.

Now multi-channel pulse amplitude analyzers have been towards digital development, and domestic and foreign about digital multi-channel pulse amplitude analyzer technology is already mature, most using FPGA constitute a system of digital circuit, and control the external analog circuit, thus improve the overall speed of the system, and reduce the power consumption of the engine, reduces the occupied volume [1] [2]. However, the design of analog terminal multichannel pulse amplitude analyzer is still applied in special industrial occasions, especially the analog multichannel pulse amplitude analyzer with low power consumption, wireless, intelligent and capable of processing large amounts of data is still being developed and used. In this paper, a portable ultra-low power multichannel pulse amplitude analyzer is designed to analyze the power consumption, input signal amplitude, spectral effect and output pulse width, amplitude, frequency and other parameters at each analog end.

\section{General Design Principle}

The principle of signal input is shown in Figure 1: the low-energy gamma signal is converted into a negative electrical signal through the action of $\mathrm{NaI}$ detector, preamplifier and high-voltage power source. After a series of analog circuits and digital MCA processing and acquisition, the energy spectrum of the radioactive source (take CS-137 as an example) within a certain period of time can be obtained (see Figure 2). The amplitude is $-247 \mathrm{mV}$, the nuclear signal is random, and the amplitude is randomly distributed [3].

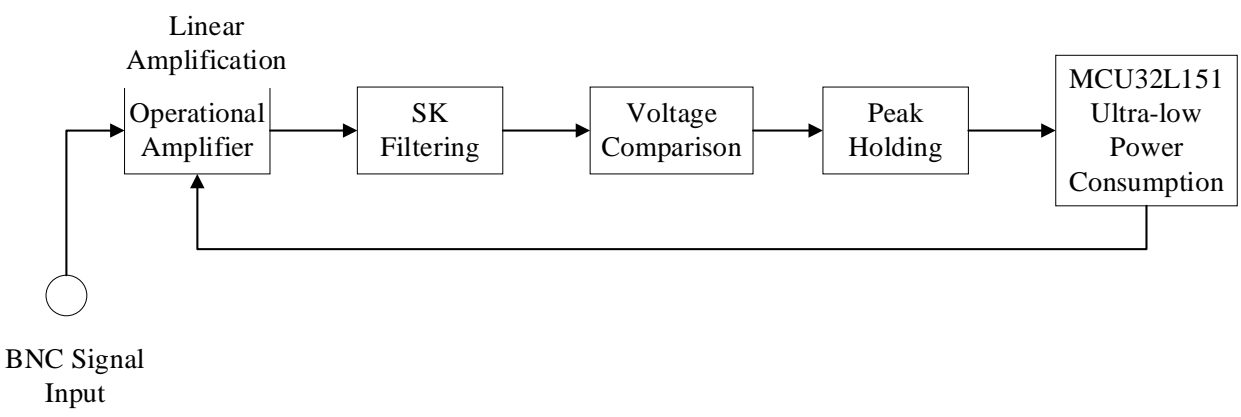

Figure 1. Schematic diagram of signal input. 


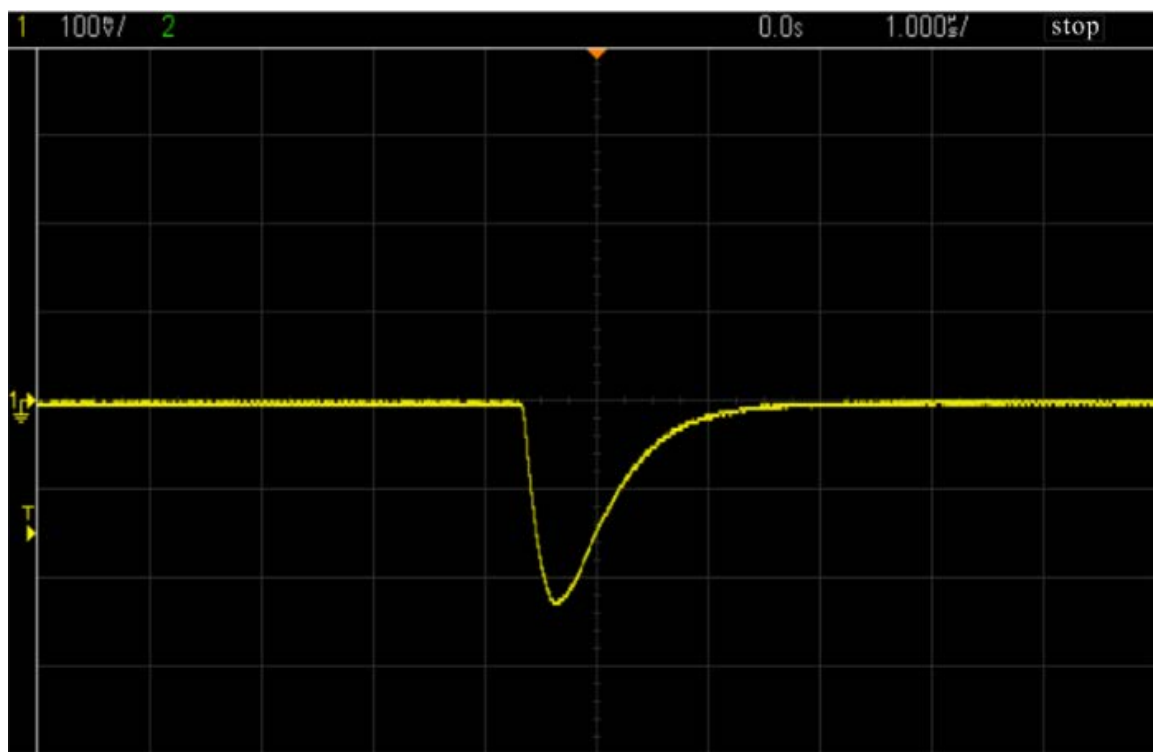

Figure 2. Original signal diagram.

During measurement, firstly input the amplitude of the input signal gradually from small to large to the input end of the amplifier and observe the amplitude of the output signal of the amplifier. When the amplitude of the input signal increases while the amplitude of the output signal does not increase, the amplifier has entered a saturation state, thus the dynamic range of the amplifier's output can be measured. As shown in Figure 3, the stability of amplification factor $\frac{\Delta A}{A}=\left.\frac{A-A^{\prime}}{A}\right|_{\Delta T}, A$ is the amplification factor under the conditions of specified temperature and set voltage. Also, only the power grid voltage of the amplifier under test is changed, and the amplification factor of the amplifier is measured. Stability of magnification $\frac{\Delta A}{A}=\left.\frac{A-A^{\prime}}{A}\right|_{\Delta V}$, Closed loop amplification factor $A=\frac{R_{f}}{R}$.

The design of this peak-hold circuit takes the sample hold chip LF398 as the core, and the ADC module's external trigger interrupt response peak time information to start $\mathrm{A} / \mathrm{D}$ conversion. $5 \mathrm{~s}$ of the monostable trigger generates $\mathrm{A} / \mathrm{D}$ conversion instruction after A/D conversion is started, and reset the peak holding circuit. The on-chip SRAM of LPC11U68 is used as the cache of energy spectrum data acquisition, and the DMA engine is used to dump A/D conversion results. The preferred ultra-low power consumption type of MCU model and ADC parameter selection are shown in Table 1.

Signal transmission is shown in Figure 4. First, it goes through the amplifier, and the gain is three times. After two stages of SK filtering and one stage of C-R circuit, the generated signal should be about $240 \mathrm{mV}$. After another voltage reformer, a drop edge signal will be generated after the two signals are different, which will be used as the AD starting signal of MCU. 


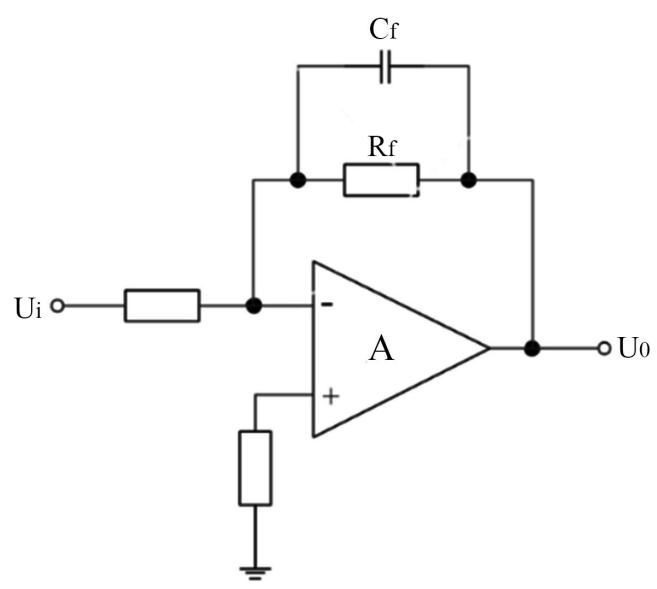

Figure 3. Schematic diagram of phase compensation method.

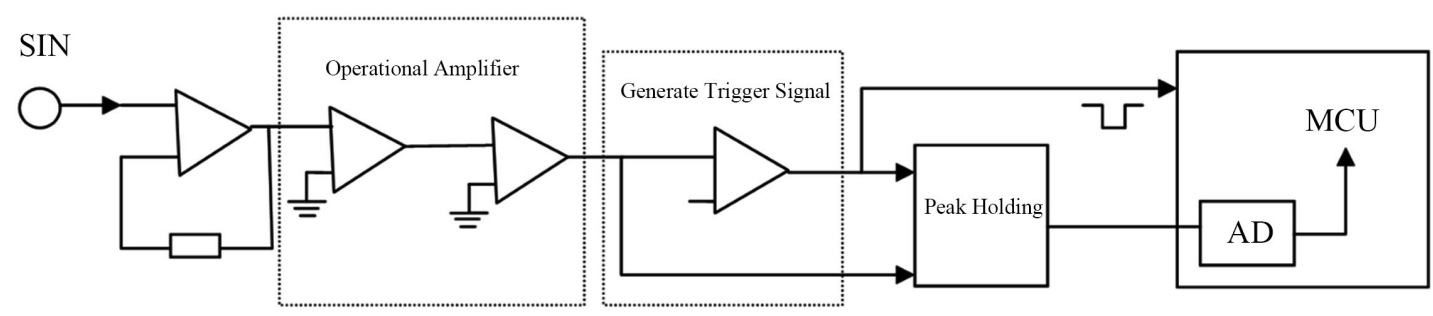

Figure 4. Schematic diagram of signal transmission.

Table 1. MCU model and ADC parameter selection.

\begin{tabular}{lllllll}
\hline MCU model & Number & ADC sample rate & Power consumption & General IO mouth & Processor core & Size \\
\hline STM32f072c8t6 & 1 & 12-bit, 1.0 $\mu \mathrm{s}$ & No low power consumption & 30 & Cortex-M0+ & $10 \mathrm{~mm} \times 10 \mathrm{~mm}$ \\
STM32L151RCT6 & 2 & $4 \mu \mathrm{s}$ & $185 \mu \mathrm{A} / \mathrm{MHz}$ & 30 & Cortex-M3 & $6.5 \mathrm{~mm} \times 6.5 \mathrm{~mm}$ \\
STM32F103ZET6 & 3 & $12 \mu \mathrm{s}$ & No low power consumption & 50 & Cortex-M3 & $15 \mathrm{~mm} \times 15 \mathrm{~mm}$ \\
MSP430FR235X & 4 & $5 \mu \mathrm{s}$ & $142 \mu \mathrm{A} / \mathrm{MHz}$ & 10 & RISC & $7 \mathrm{~mm} \times 7 \mathrm{~mm}$ \\
STM32WB55RGV6 & 5 & $12 \mathrm{bit}-25 \mathrm{~ns}$ & Activable wake up & 40 & MO+/M3 & Minimal encapsulation \\
\hline
\end{tabular}

Note: MCU (Micro Control Unit); ADC (analog to digital converter).

\section{Hardware Design}

The design of the main amplifier output circuit is shown in Figure 5. The input signal passes through the input end of the amplifier. Since the output signal of the front amplifier is a negative pulse signal, the reverse amplifier circuit is selected, and the gain is adjusted by three times. The original signal amplitude is $240 \mathrm{mV}$, the rise time is $800 \mathrm{~ns}$, a capacitor is connected in parallel at the negative feedback regulating end to maintain the voltage, and $100 \mathrm{NF}$ or $10 \mathrm{UF}$ decoupling capacitors are connected in parallel at both ends of $\pm 5 \mathrm{~V}$ to eliminate the noise caused by the digital power supply [4]. The c-R circuit at the output signal end is connected in series, which makes the signal produce downstroke and further shorten the half height and width of the signal [5].

The two-stage SK filter is designed in series with two amplifiers. As shown in 
Figure 6, the cut-off frequency of the circuit is $f_{c}=1 /(2 \pi R C)$, and the quality factor is $Q=\sqrt{C_{1} / C_{2}} / 3$ [6]. Among them, the $R=R_{1}=R_{2}=R_{3}, C=\sqrt{C_{1} C_{2}}$. Take $R=5 \mathrm{~K} \Omega, C=500 \mathrm{pf}$. SK filter circuit design as shown in Figure 7 .

For SK filter circuit debugging, test results found through two levels of SK filter and radiation signal after $c-r$ circuit (Figure 8(a)), signals with a certain down after the SK, amplitude also returned to the original signal amplitude, by comparing Figure 8(b) can see out, clearly the presence of gaussian shape signal processing results. The peak value of the signal gradually shifts to the center, and the down rush of the signal also has an obvious effect on the generation of the trigger signal of the voltage comparator behind.

After the peak hold circuit debugging, the results showed that Figure 9(a) signal for after voltage comparator, the logical end of the chip as the peak signal, the low level is as peak keep level, Figure 9(b) is after peak keep signals and trigger function comparison, we can see that after SK filtering of signal through the peak keep low level of the trigger signal to keep signals and in high level up a signal mutation; Figure 9 (c) is the comparison between the original signal graph after SK filtering and the signal after peak retention. We can see that the peak value has been maintained at about 20 US and the signal has been expanded by nearly 10 times, which can be used for in-chip $\mathrm{AD}$ acquisition under general conditions.

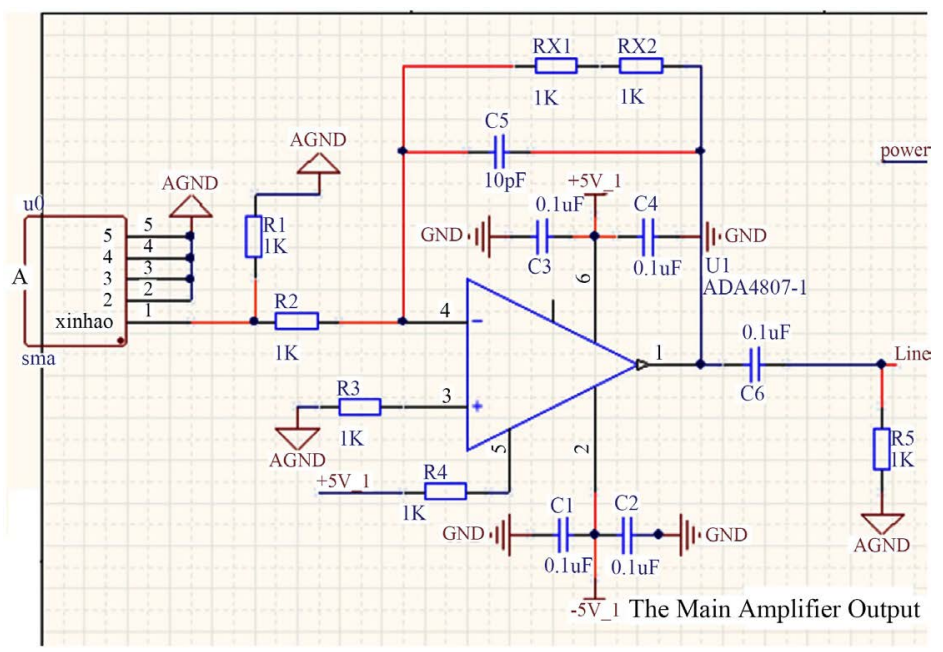

Figure 5. Schematic diagram of main amplifier output circuit.

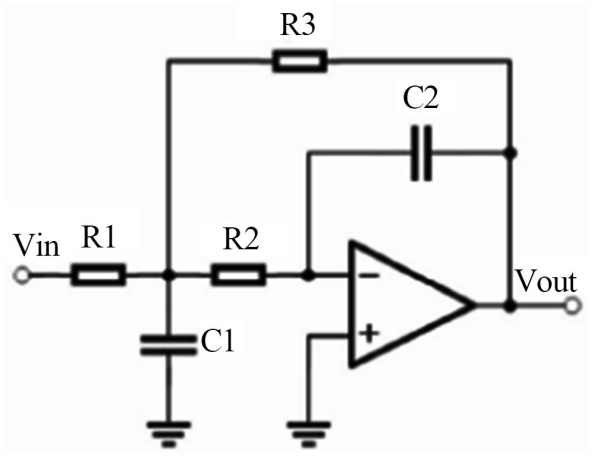

Figure 6. Schematic diagram of two-stage SK filter. 


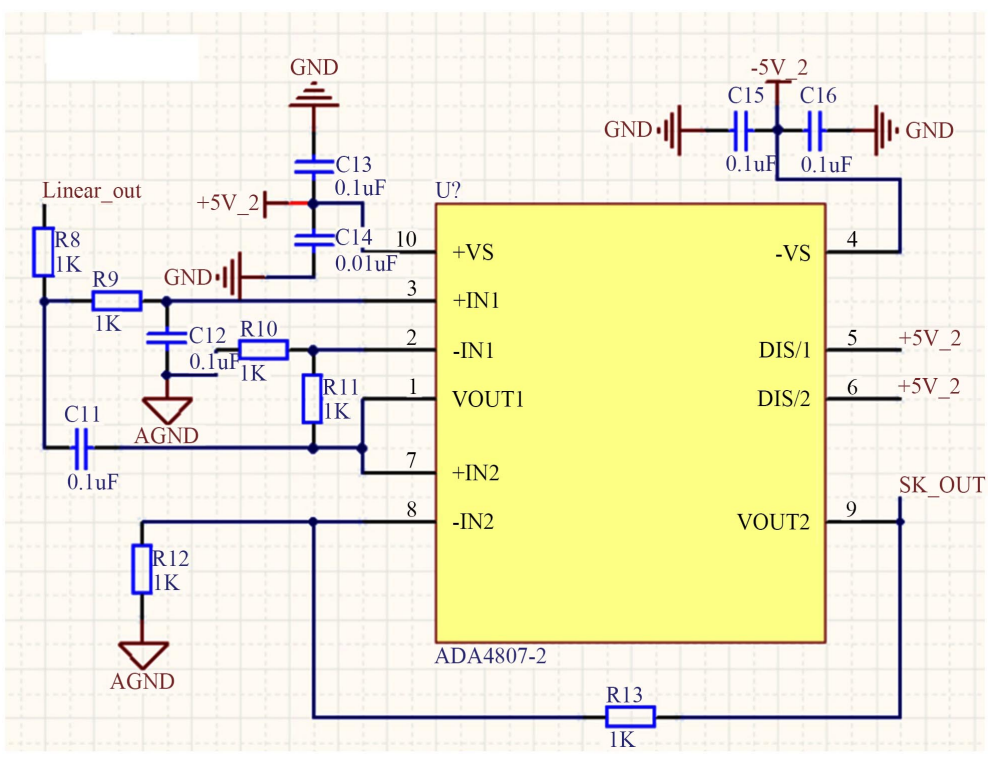

Figure 7. SK filter circuit design diagram.
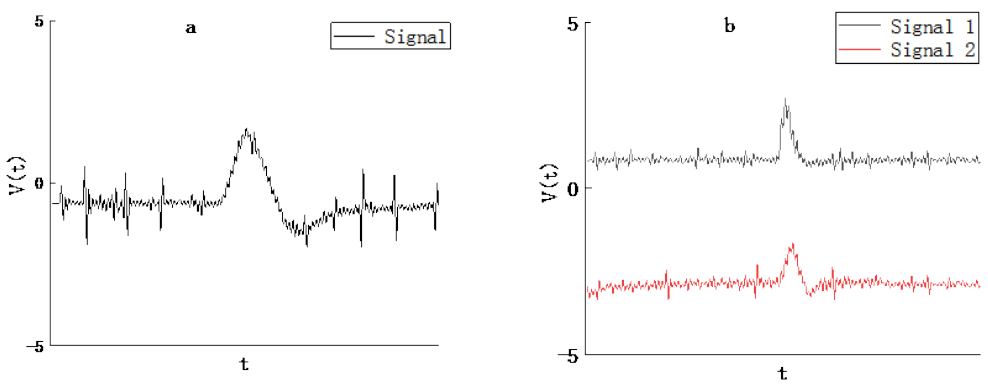

Figure 8. Comparison of SK filter circuit signal test results.
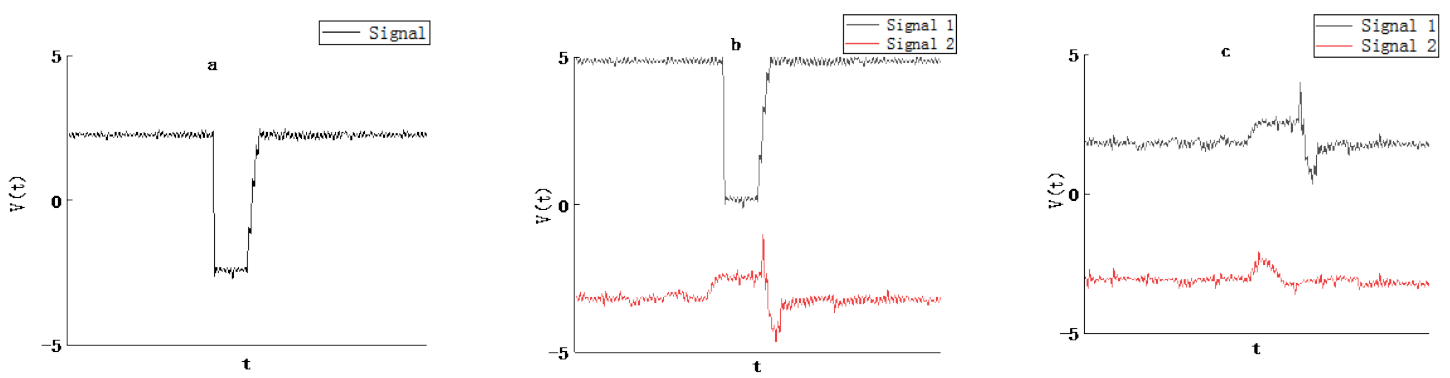

Figure 9. Voltage comparison and peak hold circuit debugging results.

\section{Conclusion}

The low-power multichannel pulse amplitude analyzer studied in this paper has a volume of only $6 \times 7 \mathrm{~cm}$, and the pulse amplitude is around $250 \mathrm{mV}$ (CS-137), with good signal stability. The design USES STM32WB55 series of dual-core chips, one chip for communication and energy spectrum collection, the other for Bluetooth communication, can achieve the acquisition of low energy gamma rays. This machine can be attached with three SPI or IIC communication devices, which can be used for the expansion of serial port and other communication methods. 
At the same time the machine uses SMA universal interface, the coaxial shielding line can carry on the nuclear data signal transmission, which has the good portability and the practicability.

\section{Acknowledgements}

Thanks to the Sichuan Key Laboratory of Geosciences and Nuclear Technology of Chengdu University of Technology for providing the platform for this project and the Sichuan Science and Technology Innovation Project for its strong support.

\section{Conflicts of Interest}

The authors declare no conflicts of interest regarding the publication of this paper.

\section{References}

[1] Ren, Y.Q. (2019) The Key Technology and System Implementation of Digital Multichannel Pulse Amplitude Analyzer Based on FPGA. MA Thesis, East China University of Technology, Nachang.

[2] Liu, H.F., Wan, Y.Q. and Tian H.Y. (2014) Design of Digital Multi-Channel Pulse Amplitude Analyzer Based on FPGA. Annual Report of China Institute of Atomic Energy, 2014.

[3] Liao, B., Tuo, X.G., Wang, H.H., et al. (2015) A Random Pulse Generator for Simulating Nuclear Radiation Signals. 2015 8th International Conference on Intelligent Computation Technology and Automation (ICICTA), Nanchang, China, 14-15 June 2015, 73-76. https://doi.org/10.1109/ICICTA.2015.27

[4] Hao. L., Zhang, Y.M. and Na, Y.X. (2013) Research on Amplification and Peak-Holding Circuits of Nanosecond light Pulse. International Symposium on Precision $\mathrm{Me}$ chanical Measurements. International Society for Optics and Photonics, 2013. https://doi.org/10.1117/12.2035894

[5] Harris, J.G., Chen, D., Wei, D., et al. (2007) Amplifier with Pulse Coded Output and Remote Signal Reconstruction from the Pulse Output.

[6] Fang, T. S. (2004) An Analytical Formula Variety of the Lower Cut-Off Frequency of Amplifier with Parallel Negative-Feedback. Journal of Xinyang Teachers College (Natural Science Edition), No. 3, 275-278. 